Int. J. Dev. Biol. 55: 11-18 (2011)

doi: $10.1387 /$ ijdb.092981sy

\title{
Ciona intestinalis Noto4 contains a phosphotyrosine interaction domain and is involved in the midline intercalation of notochord cells
}

\author{
SHIGEHIRO YAMADA* ${ }^{*}, 1,2,3$, NAOTO UENO2 ${ }^{2}$ NORI SATOH ${ }^{1,3}$ and HIROKI TAKAHASHI*,2 \\ ${ }^{1}$ Department of Zoology, Graduate School of Science, Kyoto University, Sakyo-ku, Kyoto, \\ ${ }^{2}$ Department of Developmental Biology, National Institute for Basic Biology, Okazaki, Aichi and \\ ${ }^{3}$ Marine Genomics Unit, Okinawa Institute of Science and Technology, Uruma, Okinawa, Japan
}

\begin{abstract}
Brachyury plays a pivotal role in the notochord formation in ascidian embryos. Ciona intestinalis Noto4 (Ci-Noto4) was isolated as a gene downstream of Ci-Bra. This gene encodes a 307 amino-acid protein with a C-terminal phosphotyrosine interaction domain (PTB/PID). Expression of Ci-Noto4 commences at the neural plate stage and is specific to notochord cells. Suppression of Ci-Noto4 levels with specific antisense morpholino oligonucleotides resulted in the formation of two rows of notochord cells owing to a lack of midline intercalation between the bilateral populations of progenitor cells. In contrast, overexpression of Ci-Noto4 by injection of a Ci-Bra(promoter):Ci-Noto4-EGFP construct into fertilized eggs disrupted the localization of notochord cells. Ci-Noto4 overexpression did not affect cellular differentiation in the notochord, muscle, mesenchyme, or nervous system. Analysis of Ci-Noto4 regions that are responsible for its function suggested significant roles for the PTB/PID and a central region, an area with no obvious sequence similarity to other known proteins. These results suggested that PTB/PID-containing CiNoto4 is essential for midline intercalation of notochord cells in chordate embryos.
\end{abstract}

KEY WORDS: notochord cell, morphogenetic movement, Ci-Noto4, Ci-Bra down-stream gene

\section{Introduction}

Notochord formation in ascidian embryos provides an excellent experimental system to explore cellular and molecular mechanisms involved in the development of chordate midline organs and/or the formation of chordate body plan (reviewed by Satoh, 2003; Jiang and Smith, 2007). In ascidian embryos, the notochord forms in the larval midline tail and consists of exactly 40 cells (Satoh, 1994). The lineage of these cells has been extensively detailed (Nishida, 1987). At the 110-cell stage, five pairs of progenitor cells are positioned at both right and left sides of the bilaterally symmetrical embryo. These cells converge toward the midline during gastrulation and neurulation, intercalate with each other, and extend anteroposteriorly to form a notochord consisting of a single row of cells (Miyamoto and Crowther, 1985; Munro and Odell, 2002). Later, the cells produce intracellular and/or intercellular vacuoles, which increase the volume of notochord and are required for elongation of the tail (Cloney, 1990; Jiang and Smith, 2007).

The T-box transcription factor Brachyury (Bra) plays a pivotal role in the specification of embryonic cells to a notochord fate in ascidian embryos (reviewed by Satoh, 2003; Passaneck and Di Gregorio, 2005). The upstream regulatory network of genes that leads to the transcriptional activation of the Ciona Brachyurygene (Ci-Bra) (Imai et. al., 2006; Shoguchi et al., 2008) and the genes

\footnotetext{
Abbreviations used in this paper: Ci-Bra, Ciona Brachyury gene; Ci-fibrn, Ciona fibrinogen-like gene; PID, phosphotyrosine interaction domain; PTB, phosphotyrosine binding domain.
}

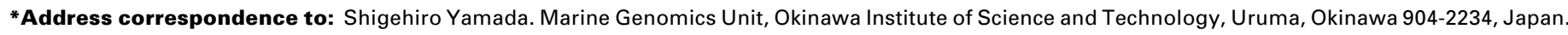

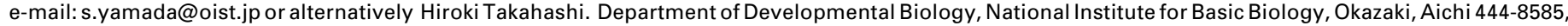
Japan.e-mail: taka@nibb.ac.jp
}

\# Present address: The Wistar Institute, 3601 Spruce Street, Philadelphia, PA 9104, USA. e-mail: syamada@wistar.org

Supplementary Material for this paper (a figure) is available at: http://dx.doi.org/10.1387/ijdb.092981sy 
downstream of $\mathrm{Ci}$-Bra that govern convergence, intercalation, extension, and differentiation of notochord cells have been extensively studied (Takahashi et al., 1999; Hotta et al., 2000; Jiang and Smith, 2007; Hotta et al., 2007; Hotta et al., 2008; Shoguchi et al., 2008; Yamada et al., 2009). Subtraction screening of normal embryos from Ci-Bra overexpressing embryos allow us to identify and characterize nearly 450 genes that are expressed in developing notochord cells directly or indirectly downstream of Ci-Bra (Takahashi et al., 1999; Hotta et al., 2000; Hotta et al., 2008). Knockdown experiments with specific antisense morpholino oligonucleotides (MO) revealed approximately 10 genes downstream of $\mathrm{Ci}$-Bra that are involved in convergent and extension movements by notochord cells (Hotta et al., 2007).

In a previous study, we examined the function of Ci-fibrn, a gene downstream of $\mathrm{Ci}$-Bra that encodes a fibrinogen-like protein (Yamada et al., 2009). Interestingly, although Ci-fibrn mRNA is restricted to notochord cells, the $\mathrm{Ci}$-fibrn protein product is distributed outside of the notochord cells in a filamentous network that resembles the notochord sheath. We previously showed that the correct distribution of Ci-fibrn was dependent on Notch signaling from the overlying neural tube (Yamada et al., 2009). In addition, Ci-fibrn interacts directly with the extracellular domain of $\mathrm{Ci}$ Notch. Aberrant Ci-fibrn distribution caused abnormal positioning of neuronal cells (Yamada et al., 2009).

In the present study, we focused on the function of another gene that is downstream of $\mathrm{Ci}-\mathrm{Bra}, \mathrm{Ci}-\mathrm{Noto} 4$, because preliminary experiments suggested that Ci-Noto4 is involved in the midline intercalation of notochord cells. Eighteen genes that were expressed in the notochord and acted downstream of $\mathrm{Ci}$-Brashowed no or weak similarity with other reported genes; we have named these genes Ci-Noto1 to Ci-Noto18(Hotta et al., 2000; Hotta et al., 2008). CiNoto4 is expressed exclusively in notochord cells beginning at the neural plate stage, and its expression persisted until the tailbud stage (see Fig. 1L of Hotta et al., 2000). CiNoto 4 cDNA consists of 1472 nucleotides that encode a 307 amino acid polypeptide (Hotta et al., 2000). We show here that $\mathrm{Ci}$-Noto4 contains a C-terminal phosphotyrosine interaction domain (PTB/PID). Proteins encoding PTB/PID function as adaptors or scaffolds that organize signaling complexes involved in a wide range of physiological processes, including neural development, immunity, tissue homeostasis, and cell growth (Uhlik et al., 2005). Although more than 200 proteins in eukaryotes and nearly 60 human proteins with PTB/PID, have been identified, to the best of our knowledge, this is the first report detailing an ascidian protein with a PTB/PID.

\section{Results and Discussion}

\section{Characterization of Ci-Noto4}

In our previous study, we showed that Ci-Noto4 cDNA consists of 1472 nucleotides with a predicted polypeptide product of 307 amino acids (Fig. 1A; Hotta et al., 2000). Moreover, Ci-Noto4 showed weak similarity to the myb-related protein PP2 from the moss Physcomitrella patens (Hotta et al., 2000). The present search for similarities between mouse proteins and Ci-Noto4 noted some homology with phosphotyrosine interaction domain containing 1 (Accession No. 98496). Notably, Ci-Noto4 contains a short PTB/PID (aa 120-135) and a C-terminal PTB/PID (aa 242299) (Fig. 1B).

\section{Functional suppression of $\mathrm{Ci}-$ Noto4 disrupted the midline alignment of notochord cells}

To examine the possible function of Ci-Noto4, we knocked down its expression levels using MO specific for Ci-Noto4. The division and movements of the notochord cells were examined after $\mathrm{Ci}$-Bra(promoter): EGFP was coinjected into fertilized eggs. Embryos that developed from eggs injected with control $\mathrm{MO}$ developed normally and contained GFP-positive notochord cells in a single row in the center of the tail by the late tailbud stage (Fig. 2A). In contrast, Ci-Noto4 MO-injected embryos did not contain a single row of notochord cells (Fig. $2 \mathrm{~B}-\mathrm{E}$ ). The progenitor cells on the left (yellow triangle) and right (white triangle) of the embryo independently converged, intercalated, and partially extended, but the left and right progenitor populations did not intercalate at the midline into a single row of cells, resulting in two rows of

\section{A 1 MHSVLLPVLYNTIIRTIPPAPKSPLAKGPGSAFDQLSGNSSIFASENRSA 50 51 EEYRQTGSFNMRRKSTSSAVSNEEMHPQHFCSPTKGDTLPLIKKPGREEK 100 101 SVLKQHAALSNTDLPRNALVNFLGSVEMSSDELRLISLERSLLTLQGCEG 150 151 RFGLLRILGDGFSLQDCNEVGAEALPVQQKNRKKKKKSGYNETEDNDDRK 200 201 PKGVPNILSCMKCVGPSRYQVLQHNNSINLKGYEVMKRKRKMIVFDYRRI 250 251 IYCGADIDGASPNLLTWLYHTVTGSYSSVQCYAVVCDDPQHARNLARAIG 300 301 QQMAALR}

B

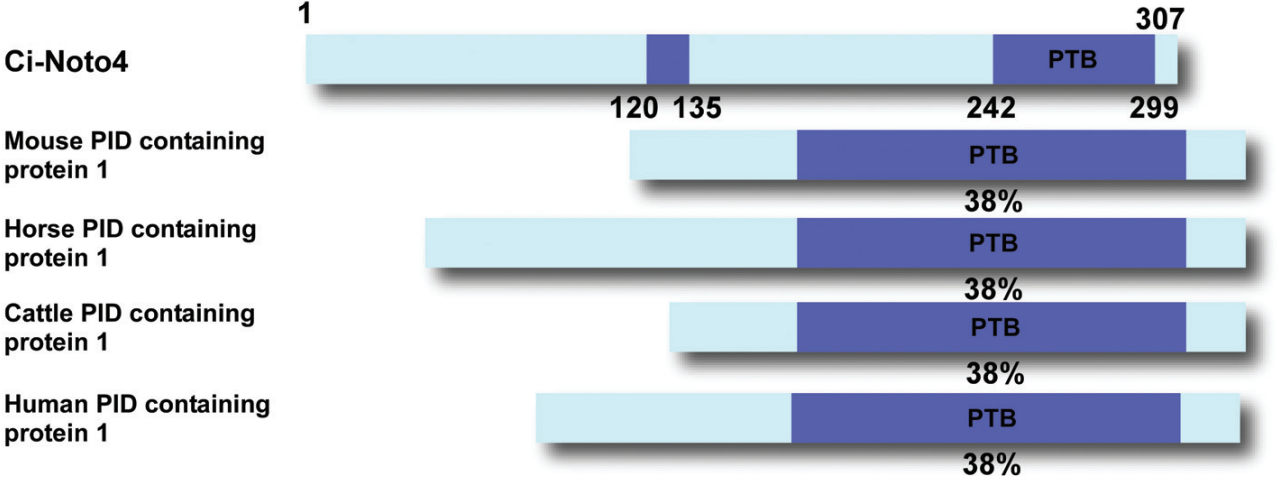

Fig. 1. Characterization of Ci-Noto4. (A) Predicted amino-acid sequence of Ci-Noto4, which consists of 307 amino acids. The short PTB/PID and C-terminal PTB/PID are denoted in blue. (B) Comparison of the amino-acid sequences of the Ci-Noto4 C-terminal PTB/PID region with those of other PTB/PID proteins. The identity (\%) of the PTB/PID amino-acid residues between Ci-Noto4 and other proteins encoding PTB/ PID is shown. 


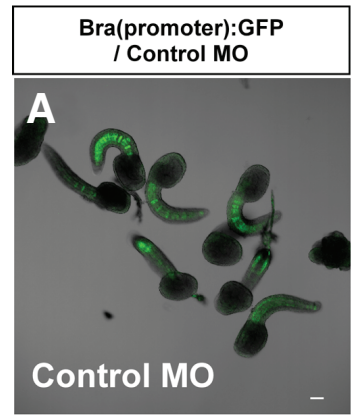

Fig. 2 (Left). Knockdown of Ci-Noto4function disrupts the alignment of notochord cells. (A) Injection of control MO did not affect notochord-cell alignment. (B-E) In contrast, injection of $\mathrm{MO}$ specific forCi-Noto4 disrupted the alignment of the noto-

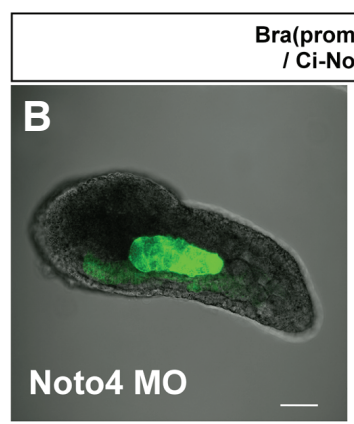

ra(promoter):GFP / Ci-Noto4 MO

\section{chord cells. (B) Some embryc}

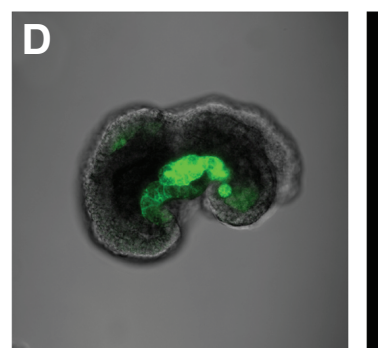

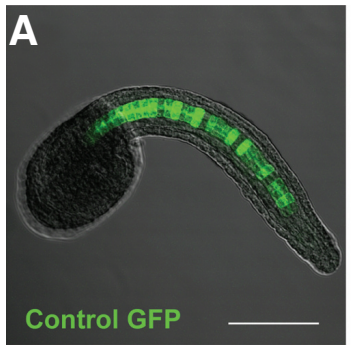
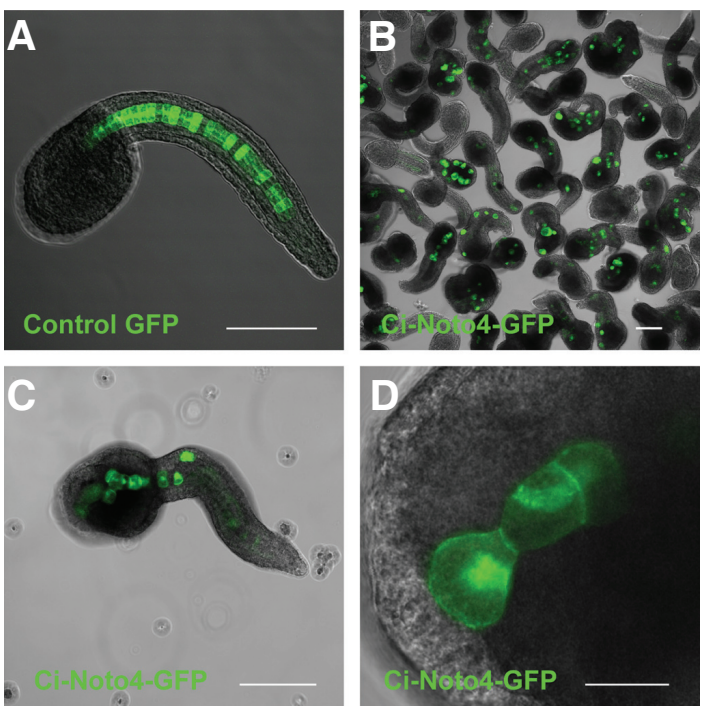

Fig. 3 (Right). Effects of Ci-Noto4 overexpression on notochord cells. Distribution of EGFP in embryos electroporated with (A) Ci-Bra(promoter):EGFP (control) or (B-D) Ci-Bra(promoter):Ci-Noto4-EGFP. (A) In control embryos, notochord cells formed a single line. Scale bar, $100 \mu$ m. (B) Ci-Noto4 overexpressing embryos showed disrupted notochord-cell alignment. Scale bar, $100 \mu \mathrm{m}$. (C) Higher magnification reveals the aberrant morphology and size of the notochord cells. The cells were not properly localized in the trunk and tail regions. Scale bar, $100 \mu$ m. (D) Higher magnification demonstrates that although Ci-Noto4-EGFP was localized in the cytoplasm of the notochord cells, strong GFP expression was observed in the nuclei. Scale bar, $20 \mu \mathrm{m}$.

notochord cells (Fig. 2 B-E).

We repeated the Ci-Noto4 MO injection experiments three times and obtained similar results. Ci-Noto4 MO injected embryos showed some range of effects on development, from embryos with normal morphology (Fig. 2 B,C) to those with damaged morphology (Fig. 2 D,E). However, most manipulated embryos showed two rows of notochord cells. Therefore, it is likely that suppression of $\mathrm{Ci}$-Noto4 expression affected the notochord cells, especially their movement, and that these notochord progenitor cells were unable to form a notochord consisting of a single row of cells.

\section{Ci-Noto4 overexpression results in notochord cells that are not properly localized in the trunk}

We examined the intracellular localization of Ci-Noto4 after electroporation of $\mathrm{Ci}$-Bra(promoter):Ci-Noto4-EGFP expression
A 1st electroporation

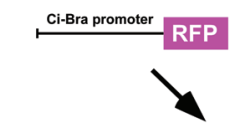

Fertilized eggs
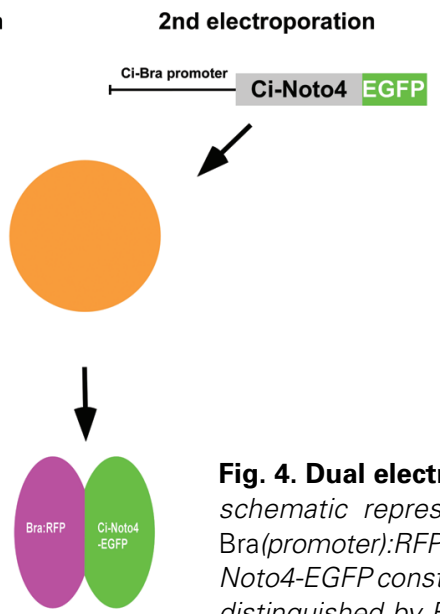

\section{Bra:RFP / Bra:EGFP}

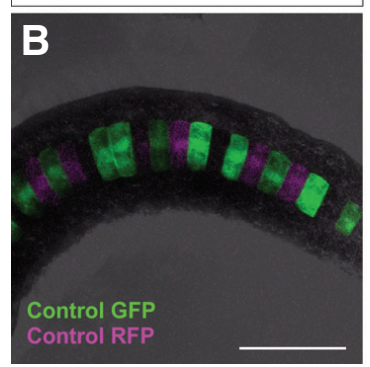

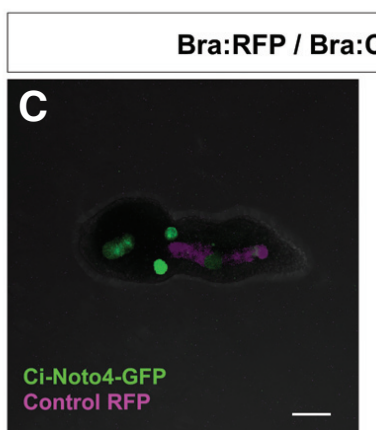

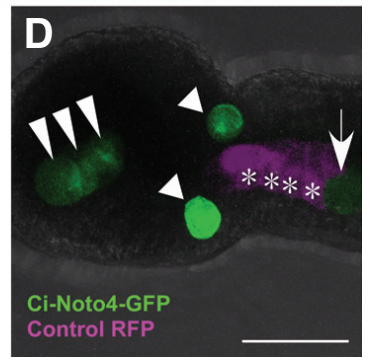

Fig. 4. Dual electroporation experiments to examine Ci-Noto4 overexpression in notochord cells. (A) $A$ schematic representation of the dual electroporation experiments. In the first electroporation, the Ci$\mathrm{Bra}$ (promoter):RFP construct was injected into fertilizedeggs. In the secondelectroporation, the Ci-Bra(promoter): $\mathrm{Ci}$ Noto4-EGFP construct was injected into the same fertilized eggs. (B) In control embryos, each notochord cell was distinguished by EGFP or RFP expression. (C,D) Ci-Noto4 overexpressing notochord cells were not properly localized in the trunk region. The morphology of the Ci-Noto4 overexpressing notochord cells also was aberrant. Magenta marks Ci-Bra(promoter):RFP expressing notochord cells. Green marks Ci-Bra(promoter):Ci-Noto4-EGFP expressing notochord cells. (D) Asterisks denote Ci-Bra(promoter):RFP expressing notochord cells. Notochord cells that strongly expressed GFP were round and localized independently (triangles). On the other hand, notochord cells with moderate levels of GFP expression were observed in clusters containing several cells (arrowheads). In contrast, notochord cells with weak levels of GFP expression were aligned with RFP-positive cells in the tail (arrow). Scale bar, $50 \mu \mathrm{m}$. 
A

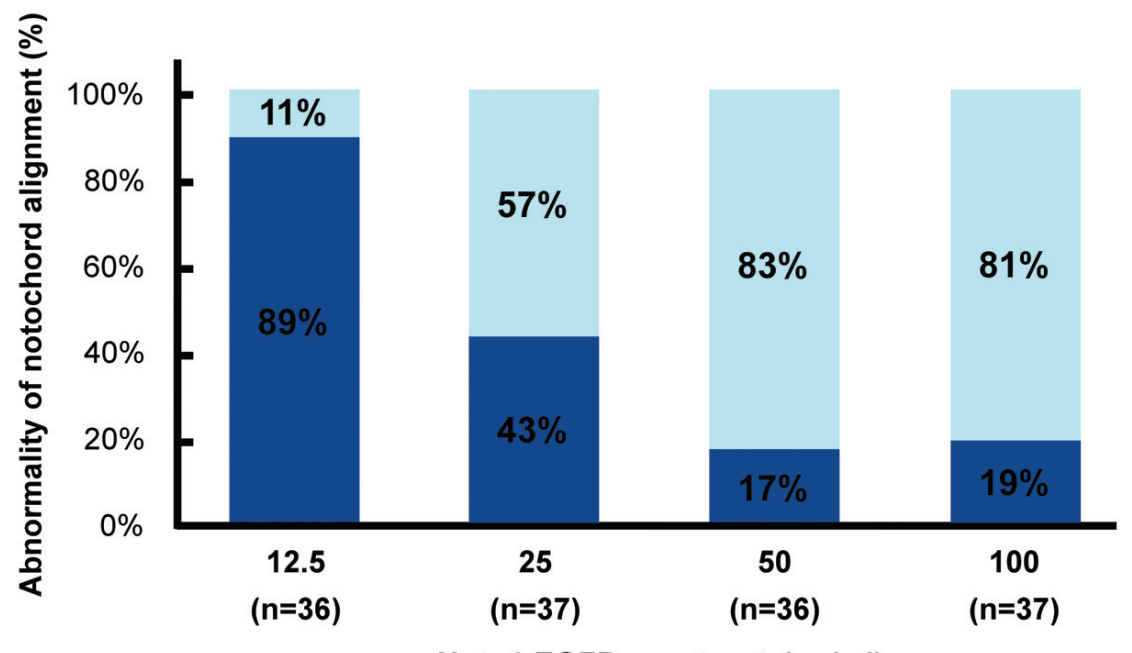

B

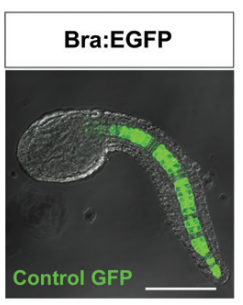

Control
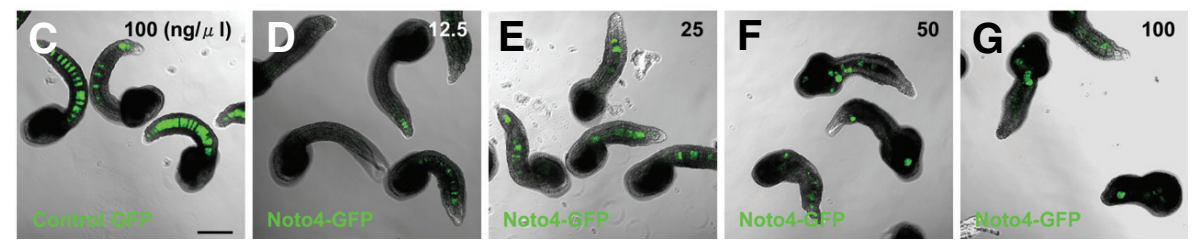

\section{Notochord Marker}
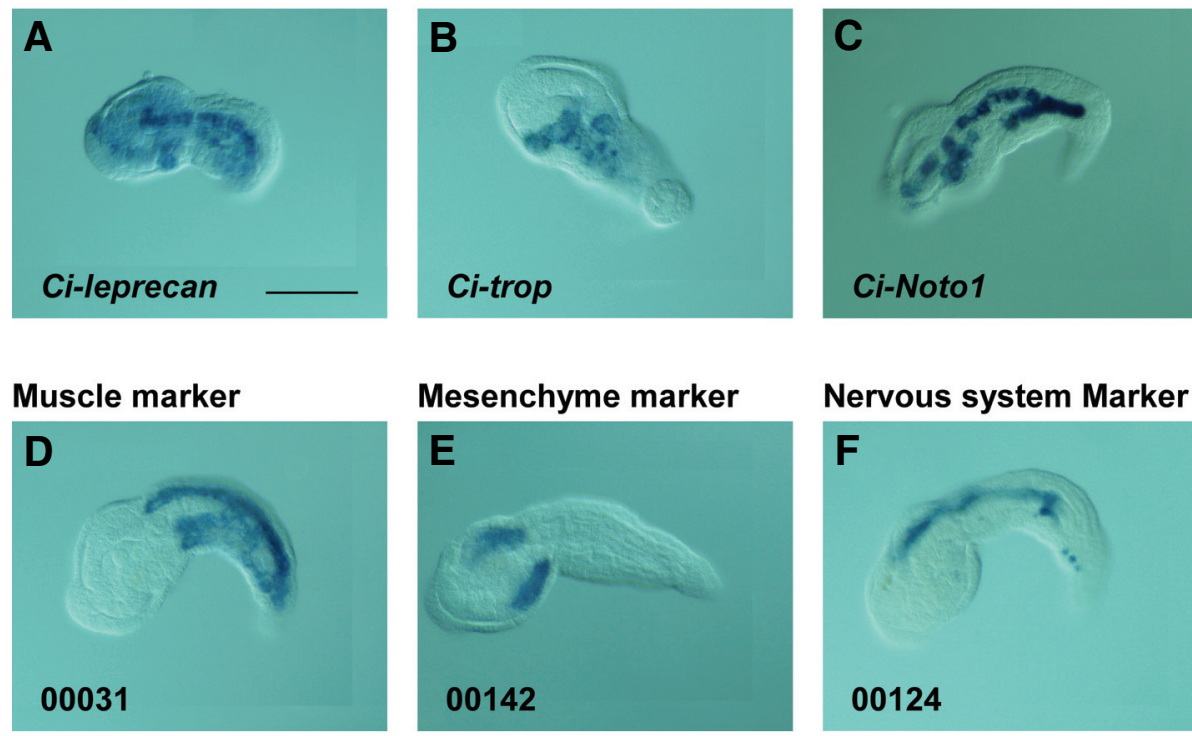

Nervous system Marker

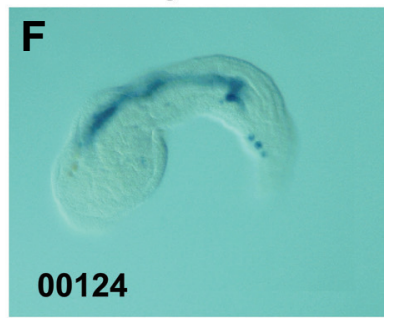

Fig. 5. Abnormal notochord cell behavior is dependent on the Ci-Noto4 levels. (A,D,E,F,G) The Ci-Bra(promoter):Noto4-EGFP construct was electroporated into fertilized eggs at four different concentrations $(12.5,25,50$ and $100 \mathrm{ng} / \mu \mathrm{l})$. (B,C) The Ci-Bra(promoter):EGFP (control) construct was electroporated into fertilized eggs at concentration of $100 \mathrm{ng} / \mathrm{\mu l}$. Ci-Noto4 overexpressing notochord cells that were properly or improperly localized in the embryos are represented by dark and light blue, respectively. $(\mathbf{A}, \mathbf{D}, \mathbf{E}, \mathbf{F}, \mathbf{G})$ The frequency of abnormal notochord alignment increased in a Ci-Noto4 dose-dependent manner. Scale bar, $100 \mu \mathrm{m}$.

constructs. GFP signal was distributed throughout the cell (see Fig. 7, panel of "low level"). Of note, we occasionally observed GFP-positive cells that were abnormally positioned (Fig. 3B). This was in contrast to normal position of GFP-positive cells in embryos injected with $\mathrm{Ci}$-Bra(promoter):EGFP (Figs. 2A and $3 \mathrm{~A}$ ). We thought that the abnormal positioning of the GFP-positive cells is due to overexpression of Ci-Noto4. More detailed analysis showed that the notochord cells of Ci-Noto4 overexpressing embryos were improperly localized in the trunk region (Fig. 3C). These cells were bigger than normal notochord cells, and at times had a spherical morphology (Fig. 3D).

However, it was possible that the observed effects were an artifact of the electroporation method to introduce $\mathrm{Ci}$ $\mathrm{Bra}$ (promoter):Ci-Noto4-EGFP rather than $\mathrm{Ci}$ Noto4 overexpression. To examine this possibility, we performed dual electroporation experiments in which two different expression constructs were separately introduced into the same embryos. We first introduced Ci-Bra(promoter):RFP (magenta) into the embryos followed by Ci-Bra(promoter):Noto4EGFP (green) (Fig. 4A). As a control, we electroporated the embryos first with $\mathrm{Ci}$ $\mathrm{Bra}$ (promoter): RFP and then with $\mathrm{Ci}$ Bra(promoter):EGFP (Fig. 4B). When the two constructs were each incorporated into opposing cells in a 2-cell embryo, a half of the notochord progenitor cells expressed GFP whereas the other half expressed RFP. As

Fig. 6. Expression of differentiation markers in Ci-Noto4 overexpressing embryos. Cell differentiation was examined using the expression of Ci-leprecan (A), Ci-trop (B), and Ci-Noto1 (C). (DF) Markers for muscle differentiation (cDNA cluster ID: 00031 (D), mesenchyme tissues (cDNA cluster ID: 00142 (E) and the nervous system (cDNA cluster ID: 00124) (F) were examined in whole mount in situ hybridizations. (A-F) Ci-Noto4 overexpression in notochord cells did not affect their cell fate. Scale bar, $50 \mu \mathrm{m}$. 
shown in Fig. 4B, dual electroporation with the control constructs did not affect embryogenesis; half of the notochord cells expressed GFP and intermingled with the other notochord cells expressing RFP, and both sets of cells were aligned in a single row. In contrast, when one of two embryonic cells received $\mathrm{Ci}$ Bra(promoter):Noto4-EGFP, the RFP expressing control cells were aligned in the midline of the tail (Fig. 4C, asterisks in D), whereas the GFP expressing cells were improperly localized in the trunk region (Fig. 4C, triangles and arrowheads in D).

Judging from the intensity of fluorescence, notochord cells with high levels of GFP expression frequently appeared round and were dispersed as single cells (Fig. 4D, triangles). Notochord cells with moderate levels of GFP expression, on the other hand, clustered in abnormal locations (Fig. 4D, arrowheads). In contrast, notochord cells with low levels of GFP expression were aligned with RFP-positive cells in the tail (Fig. 4D, arrow). These data suggest that change in the notochord cell morphology was dependent on the level of Ci-Noto4 expression within the cell.

To further examine the dose-dependent effects of Ci-Noto4 overexpression, we introduced $\mathrm{Ci}$-Bra(promoter):Ci-Noto4-EGFP into fertilized eggs by electroporation at four different concentrations $(12.5,25,50$, and $100 \mathrm{ng} / \mu \mathrm{l})$.'We counted embryos that contained abnormally located Ci-Noto4-overexpressing notochord cells. In Fig. 5, dark blue shows a normal phenotype with no dispersed notochord cells, and light blue denotes a phenotype with improperly localized Ci-Noto4overexpressing notochord cells. Injection of $12.5 \mathrm{ng} / \mu \mathrm{l}$ of the construct did not affect so much on the position of notochord cells (Fig. $5 A, D)$. Injection of 50 or $100 \mathrm{ng} / \mu \mathrm{l}$ resulted in abnormal position of notochord cells in more than $80 \%$ of manipulated embryos (Fig. 5 $A, F, G)$. The number of embryos with aberrant localization of $\mathrm{Ci}$ Noto4 overexpressing notochord cells increased in a Ci-Noto4 dosedependent manner (Fig. 5 $A, D, E, F, G$ ).

The abnormally localized notochord cells in Ci-Noto4 overexpressing embryos were examined using time-lapse video analysis. We began observing the embryos at the neurula stage under a confocal laser-scanning mi-

\section{Deletion regions of $\mathrm{Ci}-$ Noto 4}

Subcellular localization

Cell morphology
S

Spherical

S:Throughout the cell (strong GFP W:Throughout the cell (weak GFP signal in the nucleus)
307

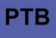

W

Normal

\section{西}
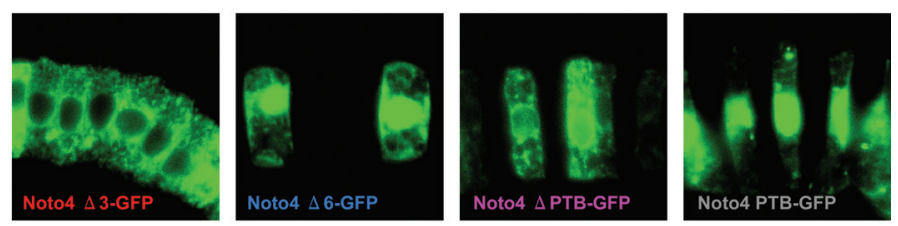

tein lacking Ci-Noto4 amino acids 24-55 showed the same in the same cell shape as were observed with high-level of full-length Noto4-EGFP expression (orange; Noto4A1, 2). A GFP fusion protein lacking Ci-Noto4 amino acids 58-201 was localized in cytoplasm and resulted in cells with a normal shape (red; Noto4A3-5). A GFP fusion protein lacking Ci-Noto4 amino acids 204-240 showed the same subcellular localization as high-level of full-length Noto4-EGFP expression, but was observed in cells with a normal morphology (blue; Noto446). A GFP fusion construct lacking the PTB/PID (amino acids 243-298) of CiNoto4 showed normal notochord cell morphology, with the protein localized throughout the cell (magenta; Noto4APTB). A GFP fusion protein lacking Ci-Noto4 amino acids 24-241 showed the same subcellular localization as high-level expression of full-length Noto4-EGFP, but resulted in normally shaped cells (gray; Noto4 PTB). Scale bars, $10 \mu \mathrm{m}$. 
croscope (Supplementary Fig. S1). The right and left progenitor cells converged toward the midline of the embryo. At the neurula stage, the cells formed a cluster. In Ci-Noto4 overexpressing embryos, notochord progenitor cells were detached from the cluster (Supplementary Fig. S1; arrowhead at $0 \mathrm{~min}$ ). These cells then stopped moving and did not converge and extend during embryogenesis (Supplementary Fig. S1, arrowheads at 0-210 $\min )$. These data suggested that the aberrant position of the CiNoto4-overexpressing notochord progenitor cells was caused by decreased movement during embryogenesis.

\section{Ci-Noto4 overexpression did not affect cellular differentia- tion}

To determine effects of Ci-Noto4 overexpression on cellular differentiation, we examined the expression of three markers of notochord differentiation: Ci-leprecan, Ci-trop, and Ci-Noto1. Each of these markers was appropriately expressed in notochord cells, suggesting that $\mathrm{Ci}$-Noto 4 overexpression did not affect the expression of notochord-specific genes (Fig. 6 A-C). We also examined the expression patterns of markers of muscle differentiation (cDNA cluster ID: 00031; Fig. 6D), mesenchyme tissue (cDNA cluster ID: 00142; Fig. 6E), and the nervous system (cDNA cluster ID: 00124; Fig. 6F). All the three markers were expressed properly (Fig. 6 D-F). These results suggest that cellular differentiation in general was not affected by Ci-Noto4 overexpression.

\section{Analysis of the functional regions in Ci-Noto4}

As mentioned above, Ci-Noto4 contains a C-terminal PTB/ PID. Several Ci-Noto4:EGFP fusion proteins that contained specific deletions in $\mathrm{Ci}$-Noto4 were employed to examine the protein regions that are responsible for its function. As a result, we identified various sequence elements that contributed to the notochord phenotypes including aberrant cell morphology and cell localization (Fig. 7).

Fusion constructs lacking amino acids 58-201 of Ci-Noto4 were localized in the cytoplasm and were associated with normal notochord cell morphology (Fig. 7 , red; Noto $4 \Delta 3, \Delta 4, \Delta 5$ ). The fusion construct lacking amino acids 204-240 of Ci-Noto4 produced normal notochord cell morphology and the same localization pattern as that was observed with high-level overexpression of full-length Ci-Noto4 (Fig. 7, blue; Noto4 $\Delta 6$ ). A fusion construct lacking the PTB/PID (amino acids 243-298) of Ci-Noto4 produced normal notochord cell morphology, with the protein localized throughout the cell, the same localization pattern as that was observed with low-level overexpression of full-length Ci-Noto4 (Fig. 7, magenta; Noto4 $\triangle \mathrm{PTB}$ ). Thus, fusion constructs lacking amino acids 58-201 or 243-298 showed altered subcellular CiNoto4 localization. Interestingly, deletion of amino acids 204-240 disrupted the function of $\mathrm{Ci}$-Noto 4 without changing the subcellular localization of the protein. These data suggested that Ci-Noto4 might play significant roles in the nucleus of the notochord cell.

Like Ci-Noto4, most phosphotyrosine-binding proteins contain a single PTB /PID without any other identifiable modular domains (Uhlik et al., 2005). Nevertheless, the central and C-terminal regions of $\mathrm{Ci}$-Noto4 also play significant roles for the function of the protein, because the deletion constructs lacking these regions did not exert the same effects as the wild-type protein when they were overexpressed under high-level expression condition.

In conclusion, Ci-Noto4 containing PTB/PID is involved in the midline intercalation of notochord cells in Ciona embryos. Loss of Ci-Noto4 function in knockdown experiments and gain of function after $\mathrm{Ci}$-Noto4 overexpression did not always result in completely different phenotypes. Rather, functional suppression produced a weak phenotype with a notochord containing two rows of cells and normal notochord cell morphology, whereas functional overexpression resulted in a strong phenotype with a complete dispersal of the individual notochord progenitor cells and spherical cell morphology. The PTB/PID and the central region play significant roles in Ci-Noto4 function. Future studies should elucidate the proteins interact with $\mathrm{Ci}-\mathrm{Noto} 4$ and how $\mathrm{Ci}$-Noto4 functions during the midline intercalation of notochord cells.

\section{Materials and Methods}

\section{Experimental design}

Ciona intestinalis adults were obtained form several places including Mikawa Bay, Gamagohri, Aichi, Maizuru Fisheries Research Station of Kyoto University, Maizuru Bay, Kyoto. Ciona intestinalis eggs and sperm were handled as described previously (Corbo et al., 1997, Mita-Miyazawa et al., 1985).

MOs were microinjected into dechorionated fertilized eggs as described below. Fertilized eggs were electroporated with"reporter constructs as previously described (Takahashi et al., 1999). Eighty-microgram aliquots of plasmid DNA were used for electroporation. After electroporation, the embryos were maintained in agar-coated dishes with Millipore-filtered seawater containing $50 \mu \mathrm{g} / \mathrm{ml}$ streptomycin sulfate (MFSWS) at $18^{\circ} \mathrm{C}$. Embryos were fixed at room temperature for $30 \mathrm{~min}$ in $4 \%$ paraformaldehyde dissolved in $0.5 \mathrm{M} \mathrm{NaCl}$ and $0.1 \mathrm{M} \mathrm{MOPS}$ at $\mathrm{pH} 7.5$. Fluorescence was observed using an LSM 510 META confocal microscope (Carl Zeiss).

\section{MO and microinjection}

MOs were made-to-order (Gene Tools). The Ci-Noto4 MO and control Ci-Noto4 5 mis MO sequences were:

5'- GGCAATAGAACTGAGTGCATGGC-3' and

5'-GGgAATAcAACTcAGTGgATcGC-3', respectively. Microinjections were carried out as described previously (Yamada et al., 2009). The control MO did not produce any marked effect.

\section{Ci-Noto4-EGFP constructs}

An 885-bp upstream sequence of $C i$-Brawas fused in-frame with DNA encoding Ci-Noto4-EGFP (monomer EGFP). This upstream sequence of $\mathrm{Ci}-\mathrm{Bra}$ contains the basal promoter and enhancers that are required for specific notochord expression. Ci-Noto4 and EGFP were fused to remove the CMV promoter from pEGFP-N1 (BD Biosciences); the vector was digested with $A$ sel and $N$ hel, blunted with Klenow fragment (Takara), and religated. To create a construct containing the $\mathrm{Ci}$-Bra promoter region (first $885 \mathrm{bp}$ upstream from the start codon) fused to EGFP, the T3 and 5'-Ci-Bra Hindll primers were used for PCR amplification. The amplified fragment was then digested with HindlII (Takara) and ligated into Hindlldigested pEGFP-N1/CMV(-). To create a construct containing Ci-Noto4 fused with EGFP, the Ci-Noto4-coding sequence was amplified by PCR. Sequences flanking Ci-Noto4 were converted to a Kozak consensus translation initiation site. The ACC Noto4 Pstl and Noto4 BamHI primers were used for PCR amplification. The amplified fragment was digested with Pstl (Takara) and BamHI (Takara) and ligated into Pstl/BamHIdigested $\mathrm{Ci}$-Bra promoter/pEGFP-N1/CMV(-). The following PCR primers were used:

T3, 5'-AATTAACCCTCACTAAAGGG-3';

5'-Ci-Bra HindllI, 5'-CCCAAGCTTCATTATAGGTTTGTAACTCG-3'; ACC Noto4 Pstl $F$,

5'-AAAACTGCAGACCATGCACTCAGTTCTATTGCCAGTGCTATAC-3'; and Noto4 BamH R, 


\section{5'-CGCGGATCCTTGCGCAATGCCGCCATCTGCTGGCCAATTGC-3'.}

\section{Dual electroporation}

The experimental scheme for dual electroporation is shown in Fig. 4A. First, the Ci-Bra(promoter):RFP construct was introduced into fertilized eggs using electroporation. Then, the Ci-Bra(promoter):Noto4-EGFP construct was immediately introduced into the same eggs. After the second electroporation procedure, embryos were raised in MFSWS at $18^{\circ} \mathrm{C}$ in agar-coated culture dishes.

\section{In vivo time-lapse imaging}

For observation, embryos were transferred to agar coated glass based dish (Iwaki glass based dishes, Asahi Techno Glass Co.) with MFSWS. Morphogenetic movements of notochord cells were followed in timelapse movies obtained using a 10x Plan-Neofluar objective lens mounted on a LSM 510 META laser scanning microscope (Carl Zeiss). Images were obtained every $2.5 \mathrm{~min}$.

\section{Ci-Noto4-EGFP expression constructs for domain analysis}

A full-length $\mathrm{Ci}$-Noto 4 construct was used as a template for PCRs. CiNoto $4 \Delta 1$ lacked amino acids 23-37. The ACC Noto4 Pstl, Del Stb1 Fu R, Del Stb1 Fu F, and Noto4 BamHI R primers were used for PCR amplification. Ci-Noto4 $\Delta 2$ lacked amino acids 23-56. The primers ACC Noto4 Pstl, Del Stb2 Fu R, Del Stb2 Fu F, and Noto4 BamHIR were used for PCR amplification. Ci-Noto4 $\triangle$ PTB lacked amino acids 242-299. The primers ACC Noto4 Pst/F, Del PTB Fu R, Del PTB Fu F, and Noto4 BamHIR were used for PCR amplification. Ci-Noto4 PTB lacked amino acids 24-241. The primers ACC Noto4 Pst/F, Noto4 1-22 PTB R, Noto4 1-22 PTB F, and Noto4 BamHI R were used for PCR amplification. Ci-Noto4 $\Delta 3$ lacked amino acids 57-116. The primers ACC Noto4 Pst/F, Noto4 Del1 R, Noto4 Del1 F, and Noto4 BamHI R were used for PCR amplification. Ci-Noto4 $\Delta 4$ lacked amino acids 87-156. The primers ACC Noto4 Pst/F, Noto4 Del2 $\mathrm{R}$, Noto4 Del2 F, and Noto4 BamHI R were used for PCR amplification. Ci-Noto4 $\Delta 5$ lacked amino acids 157-202. The primers ACC Noto4 Pst/F, Noto4 Del3 R, Noto4 Del3 F, and Noto4 BamHI R were used for PCR amplification. Ci-Noto4 $\Delta 6$ lacked amino acids 203-241. The primers ACC Noto4 PstlF, Noto4 Del4 R, Noto4 Del4 F, and Noto4 BamHIR were used for PCR amplification.

The sequences of the primers (in 5'-3' order) were as follows: Del Stb1 Fu R GTTTTCTGAAGOGAAAATGCTOGAATTTOCTITTGGOGCAGGOGGAATTGTTCGTATAATDel Stb1 Fu F ATTATACGAACAATTOOGOCTGOGOCAAAAGGAATTOGAGCATTTTCGCTTCAGAAACDel Stb2 Fu R CGTAGATTTTCTTOGCATATTAAAAGAACCTTTTGGOGCAGGOGGAATTGTTOGTATAATDel Stb2 Fu F

ATTATACGAACAATTCOGOCTGCGOCAAAAGGTTCTITTAATATGCGAAGAAAATCTACG Del PTB Fu R GCGCAATGCCGCCATCTGCTGGCCTTTCCTCTTTCGTTTCATCACTTCGTACCCDel PTB Fu F GGGTACGAAGTGATGAAACGAAAGAGGAAAGGCCAGCAGATGGCGGCATTGCGC Noto4 1-22 PTB R

CGGTAATCGAACACGATCATTTTTGGCGCAGGCGGAATTG Noto4 1-22 PTB

CAATTCCGCCTGCGCCAAAAATGATCGTGTTCGATTACCG Noto4 Del1 $R$

AAGAAGTTAACAAGCGCGTTAGTTTGCCTATACTCTTCCG Noto4 Del1 $F$ CGGAAGAGTATAGGCAAACTAACGCGCTTGTTAACTTCTT Noto4 Del2 R GAGAAACCGTCGCCGAGGATACCTTTAGTTGGAGAGCAAA Noto4 Del2 F TTTGCTCTCCAACTAAAGGTATCCTCGGCGACGGTTTCTC Noto4 Del3 R

GACAGAATATTGGGCACTCCTCGGAGCAAACCGAATCGGC Noto4 Del3 F

\section{GCCGATTCGGTTTGCTCCGAGGAGTGCCCAATATTCTGTC} Noto4 Del4 R CGGTAATCGAACACGATCATCTTGGGTTTTCGGTCATCGT Noto4 Del4 $F$ ACGATGACCGAAAACCCAAGATGATCGTGTTCGATTACCG

\section{Dose-dependent effects of Ci-Noto4 protein expression}

The Ci-Bra(promoter):Noto4-EGFP DNA construct was introduced into fertilized eggs at four different concentration (12.5, 25, 50 and $100 \mathrm{ng} /$ $\mu$ l) using electroporation. The embryos were raised in MFSWS in agarcoated culture dishes for 12 hours at $18^{\circ} \mathrm{C}$. We counted the number of embryos that contained aberrantly localized Ci-Noto4 overexpressing notochord cells.

\section{In situ hybridization}

cDNAs used were for Ci-leprecan (Hotta et al., 2000), Ci-trop (Hotta et al., 2000), Ci-Noto1 (Hotta et al., 2000), Muscle marker (cDNA cluster ID: 00031; Satou et al., 2001), Mesenchyme marker (cDNA cluster ID: 00142; Satou et al., 2001) and Nervous system marker (cDNA cluster ID: 00124; Satou et al., 2001). RNA probes were prepared with a DIG RNA labeling kit (Roche Diagnostics). Whole-mount in situ hybridizations were performed using digoxigenin-labeled antisense probes as described previously (Hotta et al., 1999). Control embryos hybridized with sense probes did not show signals above background.

\section{Acknowledgments}

We thank Dr. Chuya Shinzato for useful comment; Kazumi Taniyama and Kazuko Hirayama for technical support; Mr. Suzuki of Kaneya Seiko co., LTD for his cooperation in the equipment (ropes) of gathering the ascidians. This work was supported by Grants-in-Aid from MEXT (N.S.) and JSPS (H.T.), Japan. This study was also supported by the National Bio-Resource Project (NBRP) of MEXT/Japan, and BIRD of Japan Science and Technology Agency.

\section{References}

CLONEY, R.A., (1990). Urochordata-Ascidiacea. In Reproductive Biology of Invertebrates (Eds. K. G. Adiyodi \& R. G. Adiyodi). New Delhi: Oxford \& IBH, pp. 391451.

CORBO, J.C., LEVINE, M., ZELLER, R.W., (1997). Characterization of a notochord-specific enhancer from the Brachyury promoter region of the ascidian, Ciona intestinalis. Development 124: 589-602.

HOTTA, K., TAKAHASHI, H., ASAKURA, T., SAITOH, B., TAKATORI, N., SATOU, Y., SATOH, N., (2000). Characterization of Brachyury-downstream notochord genes in the Ciona intestinalis embryo. Dev Biol 224: 69-80.

HOTTA, K., YAMADA, S., UENO, N., SATOH, N., TAKAHASHI, H. (2007). Brachyurydownstream notochord genes and convergent extension in Ciona intestinalis. Develop Growth Differ 49: 373-382.

HOTTA, K., TAKAHASHI, H., SATOH, N., GOJOBORI, T., (2008). Brachyurydownstream gene sets in a chordate, Ciona intestinalis: Integrationg notochord specification, morphogenesis and chordate evolution. Evo Dev 10: 37-51.

IMAI, K.S., LEVINE, M., SATOH, N., SATOU, Y., (2006). Regulatory blueprint for chordate embryo. Science 312: 1183-1187.

JIANG, D., MUNRO, E.M., SMITH, W.C., (2005). Ascidian prickle regulates both mediolateral and anterior-posterior cell polarity of notochord cells. Curr Biol 15: 79-85.

JIANG, D., SMITH, W.C., (2007). Ascidian notochord morphogenesis. Dev Dyna 236: $1748-1757$

MITA-MIYAZAWA, I., IKEGAMI, S., SATOH, N., (1985). Histospecific acetylcholinesterase development in the presumptive muscle cells isolated from 16-cellstage ascidian embryos with respect to the number of DNA replication. $J$ Embryol Exp Morph 87: 1-12.

MIYAMOTO, D.M., CROWTHER, R.J., (1985). Formation of the notochord in living ascidian embryos. J Embryol Exp Morphol 86: 1-17.

MUNRO, E.M., ODELL, G.M., (2002). Polarized basolateral cell motility underlies 
invagination and convergent extension of the ascidian notochord. Development 129: 13-24.

NISHIDA, H., (1987). Cell lineage analysis in ascidian embryos by intracellular injection of a tracer enzyme. III. Up to the tissue restricted stage. Dev Biol 121: 526-541.

PASSAMANECK, Y.J., DI GREGORIO, A., (2005). Ciona intestinalis: chordate development made simple. Dev Dyn 233: 1-19.

SATOH, N. (Ed.) (1994). Developmental Biology of Ascidians. Cambridge University Press, New York.

SATOH, N., (2003). The ascidian tadpole larva: Comparative molecular development and genomics. Nat Rev Genet 4: 285-295.

SATOH, N., SATOU, Y., DAVIDSON, B., LEVINE, M., (2003). Ciona intestinalis: an emerging model for whole-genome analyses. Trends Genet 19: 376-381.

SATOU, Y., TAKATORI, N., YAMADA, L., MOCHIZUKI, Y., HAMAGUCHI, M. ISHIKAWA, H., CHIBA, S., IMAI, K., KANO, S., MURAKAMI, S. D., NAKAYAMA,
A., NISHINO, A., SASAKURA, Y., SATOH, G., SHIMOTORI, T., SHIN-I, T., SHOGUCHI, E., SUZUKI, M. M., TAKADA, N., UTSUMI, N., YOSHIDA, N. SAIGA, H., KOHARA, Y., SATOH, N., (2001). Gene expression profile in"Ciona intestinalis tailbud embryos. Development 128: 2893-2904.

SHOGUCHI, E., HAMAGUCHI, M., SATOH, N., (2008). Genome-wide network of regulatory genes for construction of a chordate embryo. Dev Biol 316: 498-509.

TAKAHASHI, H., HOTTA, K., ERIVES, A., DI GREGORIO, A., ZELLER, R.W., LEVINE, M., SATOH, N., (1999). Brachyury downstream notochord differentiation in the ascidian embryo. Genes Dev 13: 1519-1523.

UHLIK, M.T., TEMPLE, B., BENCHARIT, S., KIMPLE, A. J., SIDEROVSKI, D. P. JOHNSON, G. L., (2005). Structural and evolutionary division of phosphotyrosine binding (PTB) domains. J Mol Biol 345: 1-20.

YAMADA, S., HOTTA, K., YAMAMOTO, T.S., UENO, N., SATOH, N., TAKAHASHI, H., (2009). Interaction of notochord-derived fibrinogen-like protein with Notch regulates the patterning of the central nervous system of Ciona intestinals embryos. Dev Biol 328: 1-12.

\section{Further Related Reading, published previously in the Int. J. Dev. Biol.}

See our recent Special Issue Placenta edited by Joan S. Hunt and Kent L. Thornburg at:

http://www.ijdb.ehu.es/web/contents.php?vol=54\&issue=2-3

$\mathrm{XSu}(\mathrm{H}) 2$ is an essential factor for gene expression and morphogenesis of the Xenopus gastrula embryo

Motoaki Ito, Tomohisa Katada, Seiji Miyatani and Tsutomu Kinoshita

Int. J. Dev. Biol. (2007) 51: 27-36

Synergistic action of HNF-3 and Brachyury in the notochord differentiation of ascidian embryos.

Y Shimauchi, S Chiba and N Satoh

Int. J. Dev. Biol. (2001) 45: 643-652

Characterization of an ascidian maternal T-box gene, As-mT.

N Takada, K Tagawa, H Takahashi and N Satoh

Int. J. Dev. Biol. (1998) 42: 1093-1100

HrWnt-5: a maternally expressed ascidian Wnt gene with posterior localization in early embryos.

Y Sasakura, M Ogasawara and K W Makabe

Int. J. Dev. Biol. (1998) 42: 573-579

Alterations in gene expression during mesoderm formation and axial patterning in Brachyury (T) embryos.

P Rashbass, V Wilson, B Rosen and R S Beddington

Int. J. Dev. Biol. (1994) 38: 35-44

5 yr ISI Impact Factor $(2008)=3.271$

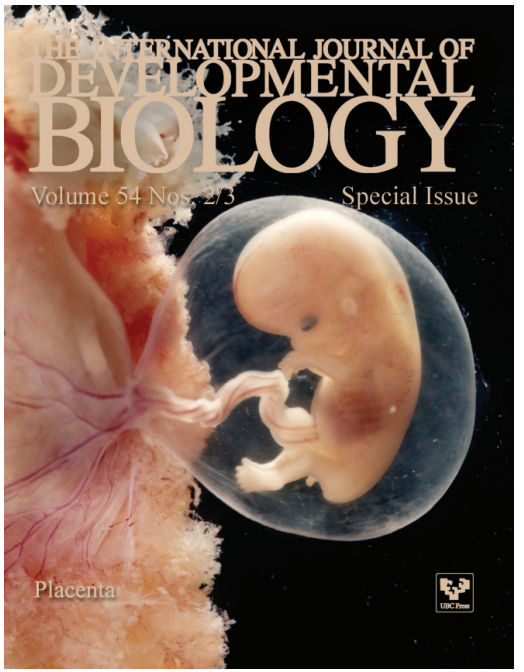

